\title{
Merkmal(s?)analyse, Seminar(s?)arbeit und Essen(s?)ausgabe: Zweifelsfälle der Verfugung als Indikatoren für Sprachwandel ${ }^{\star}$
}

\author{
DAMARIS NÜBLING \& RENATA SZCZEPANIAK
}

\section{Abstract}

Contemporary German abounds in doubtful cases where linking elements alternate with zero elements, such as Seminar $(+\mathrm{s}$ ?+)arbeit 'term paper', Respekt $(+\mathrm{s} ?+)$ person 'person who commands respect". This variation indicates a profound language change in the course of which the linking $+\mathrm{s}+$ has spread continuously since Early New High German and is replacing the zero element more and more often. Today, $+\mathrm{s}+$ is the most productive, progressive and most frequently occurring linking element. In this paper, we provide an explanation for the doubtful cases. Most often, the linking $+\mathrm{s}+$ depends directly on the phonological quality of the first part of the compound: the worse its phonological structure, the more likely the occurrence of the linking $+\mathrm{s}+$. It occurs most regularly after first parts of compounds containing a suffix or an unstressed prefix (Verkáuf $+\mathrm{s}+$ gespräch 'sales conversation'), while words with an ideal phonological structure (monosyllabic or trochaic words) rarely attract the linking $+\mathrm{s}+$. The variation concentrates on compounds whose first parts feature a stressed prefix (Éinkauf $(+\mathrm{s} ?+)$ führer 'shopping guide'). There is, however, a further factor which leads to fluctuation in the occurrence of the linking $+\mathrm{s}+$. In cases where the second part of synthetic compounds such as Auftrag $(+\mathrm{s} ?+)$ geber 'client' contain a high degree of verbality, the linking $+\mathrm{s}+$ blurs the syntactic relation between the immediate constituents, strengthening the morphological character of the compound.

Keywords: linking elements, language change, word formation, compounds, doubtful cases.

* Dieser Beitrag ist im Rahmen des Mainzer Forschungsprojekts "Determinanten sprachlicher Variation" entstanden. 


\section{Das Problem}

Aktueller Sprachwandel manifestiert sich immer in Unsicherheiten, die, wenn sie von den Sprechern reflektiert werden, als Zweifelsfälle bezeichnet werden (Klein 2003). Fugenelemente sind derzeit besonders stark von solchen Schwankungen betroffen, z. B. Respekt + s? + person, Seminar $+s ?+$ arbeit, Dreieck $+s ?+$ tuch . Verschiedene Sprachberatungsstellen berichten übereinstimmend, dass unter den wortbildungsmorphologischen Anfragen Probleme der Verfugung den größten Anteil stellen, so etwa die Duden-Sprachberatungsstelle, denen wir die meisten Zweifelsfälle, die in diesem Beitrag analysiert werden, verdanken. ${ }^{1}$ Auch die Sprachberatungsstelle der Gesellschaft für deutsche Sprache in Wiesbaden und andere Einrichtungen äußern Ähnliches (s. Mackowiak \& Steffen 1991: 522, Frilling 2004: 47, 2005: 75, Strobel 2008: 38). Bei diesen Schwankungsfällen handelt es sich um Fremdwörter (Interessen $+s ?+$ konflikt) wie um Erbwörter $($ Seehund $+s ?+$ jagd $)$, um Alltagswörter $($ Schiff $+s ?+-$ reise) wie um Fachwortschatz (Schaden + s? + ersatz). Zur Notation: Das Fugenelement (verkürzt auch: Fuge) wird immer durch ' + ' vom Erstund Zweitglied getrennt.

Ein sprachlicher Zweifelsfall ist nach der Definition von Klein "eine sprachliche Einheit (Wort/Wortform/Satz), bei der kompetente Sprecher im Blick auf (mindestens) zwei Varianten $(\mathrm{a}, \mathrm{b} \ldots$...) in Zweifel geraten können, welche der beiden Formen (standardsprachlich) korrekt ist" (Klein 2003: 2). Es werden dabei keine Prozentangaben für Schwankungen in Texten genannt, d. h. für einen Zweifelsfall reicht das Faktum des Zweifelns, und dies dürfte gegeben sein, wenn Sprachbenutzer sich mit solchen Fällen an Sprachberatungsstellen wenden. Dennoch werden wir Korpusstichproben nehmen.

Auffällig ist, dass es sich bei den Zweifelsfällen fast ausschließlich um Probleme der $s$-Fuge handelt: Immerhin gibt es, die sog. Nullfuge (Nichtverfugung) und die sog. subtraktive Fuge (Miete - Mietpreis) nicht mitgezählt, sieben verschiedene Fugenelemente, darunter, neben $-s$ - als dem häufigsten Element, auch -(e)n- (Frau+en+schuh, Blume+n+topf),-es$($ Land $+e s+k i n d e r)$ und -er- $($ Kind $+e r+s c h u h)$ (zu Näherem s. Augst 1975, Ortner et al. 1991, Becker 1992, Fuhrhop 1996, 1998, 2000, Eisenberg 2006, Nübling \& Szczepaniak 2008). In Tabelle 1 listen wir in alphabetischer Ordnung die typischen, oft mehrfach nachgefragten Zweifelsfälle auf, abgesehen von den (nicht seltenen) Beispielen, in denen das fragliche Fugen- $s$ von einem mit [s, z] anlautenden Kompositionsglied gefolgt wird, denn hier ist nicht klar entscheidbar, ob es sich nur um ein

1. Hierfür möchten wir uns sehr bei Yvonne Goldammer, Franziska Münzberg und Melanie Kunkel bedanken. 
Tabelle 1: Zweifelsfälle der Verfugung im gegenwärtigen Deutsch.

\begin{tabular}{|r|l|}
\hline Nr. & Zweifelsfall \\
\hline 1. & Abitur $(s)$ feier \\
\hline 2. & Advent $(s)$ abend \\
\hline 3. & Analyse $(n)$ ergebnis \\
\hline 4. & Anfahrt $(s)$ beschreibung \\
\hline 5. & Antrag $(s)$ steller \\
\hline 6. & Anwalt $(s)$ beruf \\
\hline 7. & Arbeit $(s)$ nehmer \\
\hline 8. & Aufwand $(s)$ entwicklung \\
\hline 9. & Aufzug $(s)$ vorrichtung \\
\hline 10. & Ausschlag $(s)$ richtung \\
\hline 11. & Beirat $(s)$ vertretung \\
\hline 12. & Bestand $(s)$ schutz \\
\hline 13. & Datum $(s)$ angabe \\
\hline 14. & Denkmal $(s)$ pflege \\
\hline 15. & Diebstahl $(s)$ versicherung \\
\hline 16. & Diplom $(s)$ feier \\
\hline 17. & Dreieck $(s)$ tuch \\
\hline 18. & Einkauf $(s)$ führer \\
\hline 19. & Einkommen $(s)$ steuer \\
\hline 20. & Erbschaft $(s)$ steuer \\
\hline 21. & Essen $(s)$ ausgabe \\
\hline 22. & Gesang $(s)$ verein \\
\hline 23. & Gesang $(s)$ buch \\
\hline 24. & Gewicht $(s)$ heber \\
\hline 25. & Handwerk $(s)$ gipfel \\
\hline 26. & Interessen $(s)$ konflikt \\
\hline 27. & Kont $($ olen $)$ führung \\
\hline
\end{tabular}

\begin{tabular}{|c|c|}
\hline Nr. & Zweifelsfall \\
\hline 28. & Knochenmark $(s)$ transplantation \\
\hline 29. & Krieg $(s)$ führung \\
\hline 30. & Latinum $(s)$ kurs \\
\hline 31. & Lehramt $(s)$ kandidat \\
\hline 32. & Mode (n)schau \\
\hline 33. & Mehrwert(s)steuer \\
\hline 34. & Miet $(s)$ haus \\
\hline 35. & Mitglied $(s)$ staat \\
\hline 36. & $\operatorname{Praktikum}(\mathrm{s})$ platz \\
\hline 37. & Rechnung $(s)$ stellung \\
\hline 38. & Referat $(s)$ besprechung \\
\hline 39. & Respekt(s)person \\
\hline 40. & Sachstand(s)anfrage \\
\hline 41. & Schaden $($ s)ersatz \\
\hline 42. & Schiff $($ s) reise \\
\hline 43. & Schifffahrt (s)unfall \\
\hline 44. & Seminar (s)arbeit \\
\hline 45. & Seehund (s)jagd \\
\hline 46. & Sichtvermerk $(s)$ zwang \\
\hline 47. & Sorgerecht $(s)$ prozess \\
\hline 48. & Stellung (s)nahme \\
\hline 49. & Testament $(s)$ eröffnung \\
\hline 50. & Umbruch $(s)$ geschichte \\
\hline 51. & Unterschrift(en/s)aktion \\
\hline 52. & Verband $($ s) kasten \\
\hline 53. & Widerrufsrecht(s)belehrung \\
\hline 54. & Werk $(s)$ leiter \\
\hline
\end{tabular}

akustisches Problem handelt (das Fugen- $s$ assimiliert mit dem Folge- $s$ ), aus dem ein graphisches Umsetzungsproblem resultiert, z. B. $A d$ vent $(+s+)$ singen, Antrieb $(+s+)$ system, Aschermittwoch $(+s+)$ sitzung. Allerdings haben wir in unserem Korpus solche Fälle belassen, deren zweites Kompositionsglied mit palatalem [J] anlautet, da sich dessen Artikulationsort von dem eines [s] bzw. [z] unterscheidet (z.B. Bestand $(+s+)$ schutz, Einkommen $(+s+)$ steuer $)$. 
Tabelle 2: Zweifelsfälle der Verfugung bei linguistischen Termini.

\begin{tabular}{|l|l|}
\hline Nr. & Zweifelsfall \\
\hline 1. & Ablaut $(s)$ reihe \\
\hline 2. & Auslaut $(s)$ verhärtung \\
\hline 3. & Festland $(s)$ dialekte \\
\hline 4. & Merkmal $($ s) analyse \\
\hline 5. & Namen $(s)$ forschung \\
\hline
\end{tabular}

\begin{tabular}{|r|l|}
\hline Nr. & Zweifelsfall \\
\hline 6. & Präteritum $($ s) schwund \\
\hline 7. & Sprachstand $($ s) stufen \\
\hline 8. & Sprachwandel $($ s) phänomen \\
\hline 9. & Subjekt $($ s) pronomen \\
\hline 10. & Umlaut $($ s) bezeichnung \\
\hline
\end{tabular}

Öfter hat ein und dasselbe Erstglied mit schwankender $s$-Verfugung je nach folgendem Zweitglied zu mehreren Anfragen geführt, z. B. Praktikum $(+s+)$ platz, -stelle, -bericht, Werk $(+s+)$ leiter, -auffahrt. Aus Gründen der Übersichtlichkeit haben wir solche Mehrfachnennungen zu nur einem Kompositum zusammengefasst, auch wenn es, wofür wir in Abschnitt 3.1 argumentieren, seitens des Zweitglieds Einflüsse auf das Fugenverhalten gibt. Der Zweifelsfallcharakter all dieser Beispiele lässt sich schnell durch eine Google- bzw. Cosmas-Recherche bestätigen. Die folgende Zusammenstellung ist bei weitem nicht vollständig: Die derzeit existierenden Zweifelsfälle dürften in die Hunderte gehen.

Tabelle 2 fügt eine eigene Sammlung frequenter fachsprachlich-linguistischer Komposita mit Verfugungsschwankungen hinzu, die in unsere spätere Analyse mit einbezogen werden.

Die überwiegende Mehrzahl der Zweifelsfälle betrifft die Alternative Null- vs. $s$-Verfugung. Die wenigen anderen betreffen die (e)n-Fuge und sind in Tabelle 1 grau hinterlegt: Analyse $(+n+)$ ergebnis, Kont $(+o+1$ + en + führung, Mode $(+n+)$ schau und Unterschrift $(+s+/+$ en +$)$ aktion. Bei diesen Fällen dürfte eine pluralische Lesart der (e)n-Fuge die Schwankung motivieren, wenngleich psycholinguistischen Untersuchungen zufolge Fugenelemente weder Numerus- noch Kasusmarker darstellen (Dressler et al. 2001, Koester et al. 2004). Dennoch kommt es bei vielen Muttersprachlern $\mathrm{zu}$ solchen Interpretationen ("es muss doch Unterschriften- und nicht Unterschriftsaktion heißen, da viele Unterschriften eingeholt werden"). ${ }^{2}$ Tatsächlich ist eine pluralische Lesart der

2. Schwankende Fugenelemente werden öfter reflektiert und dann auch meist "reflexiviert" (remotiviert) in dem Sinn, dass man ihnen eine flexionsmorphologische Funktion unterstellt, d.h. sie als Flexive reanalysiert. $\mathrm{Zu}$ den ausgeprägten Schwankungsfällen gehört auch Namen $(+s+)$-, vgl. Namen $(+s ?+)$ kunde, Namen $(+s ?+)$ forschung etc. (hierzu s. Nübling 2004). So plädiert Brendler (2004: 45) für eine terminologische Funktionalisierung der Fugenelemente: Namen $\varnothing+$-Komposita sollen (pluralisch) Einzelnamen in ihrer Vielheit bezeichnen (daher z. B. Namen $+\varnothing+k u n d e$ ), Namen $+s+-K o m p o-$ sita dagegen nur die abstrakte Kategorie des Eigennamens (daher z. B. Namen+s+theorie). 
en-Fuge nicht immer auszuschließen, gerade bei schwachen Feminina ohne nachtonige Reduktionssilbe wie Unterschrift, Geburt, weshalb es wenn, dann genau hier zu entsprechenden Problemen kommt. Auch den drei anderen Schwankungsfällen dürfte die erwünschte Plurallesart zugrunde liegen. Bei Konto und Analyse handelt es sich außerdem um Fremdwörter, deren Fugenverhalten ohnehin oft schwankt (vgl. Augst 1975, Nübling \& Szczepaniak 2009). Wir werden diese vier Fälle im Folgenden ausschließen, da wir uns nur auf den prototypischen Schwankungsfall der Null- vs. $s$-Fuge konzentrieren, der eine wichtige Etappe im Sprachwandel markiert.

Die Frage ist, ob die Entwicklung zur Null- oder zur $s$-Verfugung hin verläuft. U.E. handelt es sich bei der $s$-Fuge um den progressiven Fall, der die Schwankung verursacht, d.h. bisher nichtverfugte Komposita nehmen immer öfter die $s$-Fuge. Hiergegen sprechen nur wenige Fälle aus dem juristischen Fachwortschatz. So sind Komposita auf -schaft nach allen bekannten Regeln $s$-verfugt (Mannschaft $+s+$ spiel, Bekanntschaft $+s+$ grad etc.) - mit Ausnahme des Zweifelsfalls Erbschaft $(+s+)$ steuer: Nur im juristischen Fachwortschatz werden Fugenelemente unterdrückt, die in vergleichbaren Fällen fraglos aufträten (vgl. auch Scha$\operatorname{den}(+s+)$ ersatz, Mehrwert $(+s+)$ steuer, Sorgerecht $(+s+)$ prozess $){ }^{3}$ In anderen Fällen hat sich die $s$-Fuge langfristig nicht durchgesetzt, gerade bei manchen linguistischen Fachwörtern wie Ablaut $(+s+)$ reihe, Auslaut $(+s+)$ verhärtung, Präteritum $(+s+)$ schwund. Hier entstammen die Beispiele älteren linguistischen Darstellungen. Häufig finden sich die Verfugungen auch in studentischen Hausarbeiten, d.h. die Schwankung ist historisch nicht abgeschlossen. In der linguistischen Fachsprache durchgesetzt haben sie sich indessen (noch) nicht, vielleicht aus ähnlichen Gründen wie beim juristischen Fachwortschatz. In allen anderen Fällen dürfte auf der Hand liegen, dass der Trend in Richtung $s$-Fuge geht. Dass die $s$-Fuge sekundär und die Nullfuge primär ist, zeigen sämtliche unparadigmischen Vorkommen von $-s-$ (d.h. im betreffenden Flexionsparadigma kommt sonst keine $s$-Endung vor). Michel (2010) legt eine Studie zum Frühneuhochdeutschen vor (hier bzgl. des Zeitraums 13501600), in der er die insgesamt $2.555 \mathrm{~N}+\mathrm{N}$-Komposita im Kleinen Frühneuhochdeutschen Wörterbuch von Baufeld (1996) extrahiert und sie auf ihr Fugenverhalten hin analysiert hat. Dabei sind $87 \%$ unverfugt (heute: $57,6 \%$ ), knapp $10 \%$ (e)n-verfugt (heute: $13 \%$ ) und nur 2,3\% s-verfugt

3. Hierzu das Duden-Wörterbuch der sprachlichen Zweifelsfälle (2007: 336): "Durch behördliche Sprachregelung ist in diesen Komposita [solchen auf -steuer - D.N./R.S.] das Fugen-s getilgt worden: Einkommen-, Grunderwerb-, Körperschaft-, Vermögen-, Versicherungsteuer u. a. Neben diesem amtlichen Gebrauch sind aber Formen mit Fugen-s (Einkommenssteuer usw.) üblich und berechtigt". 
(heute: $25 \%$ ). Damit lässt sich nachweisen, dass die $s$-Fuge einen steilen Aufstieg erfahren hat. ${ }^{4}$ Im gesamten Korpus zum Frühnhd. befinden sich nur zwei $s$-verfugte Feminina im Erstglied, d.h. unparadigmische $s$-Fugen treten noch fast nicht auf. Michel (2009) geht auf die zunehmende $s$ - statt Nullverfugung von Schwankungsfällen wie Schaden $+s+-$ ersatz (s. auch Diagramm 3 unten) und Einkommen + s + steuer ein und begründet die häufiger werdende $s$-Fuge mit deren diaphasischer Unmarkiertheit. Umgekehrt weiß man, dass sich unter den $25 \% s$-verfugter Komposita im Nhd. mehrheitlich unparadigmische $s$-Fugen befinden (Ortner et al. 1991: 76). Um auch für die heutige Zeit Evidenz dafür zu liefern, dass die $s$-Fuge sich weiterhin gegen die Nullfuge durchsetzt, haben wir eine Recherche im DWDS-Korpus vorgenommen. Die meisten Anfragen zu unseren Zweifelsfällen ergaben nur sehr wenige Treffer, oft im einstelligen Bereich, doch zeigen diejenigen mit mehr Treffern eine deutliche Tendenz zur $s$-Fuge im diachronen Verlauf, s. die Diagramme 1-3. - Diagramm 1 zeigt, dass seit 1940 die $s$-Verfugung bei Arbeitsloser die Regel darstellt (heute handelt es sich nicht mehr um einen Schwankungsfall).

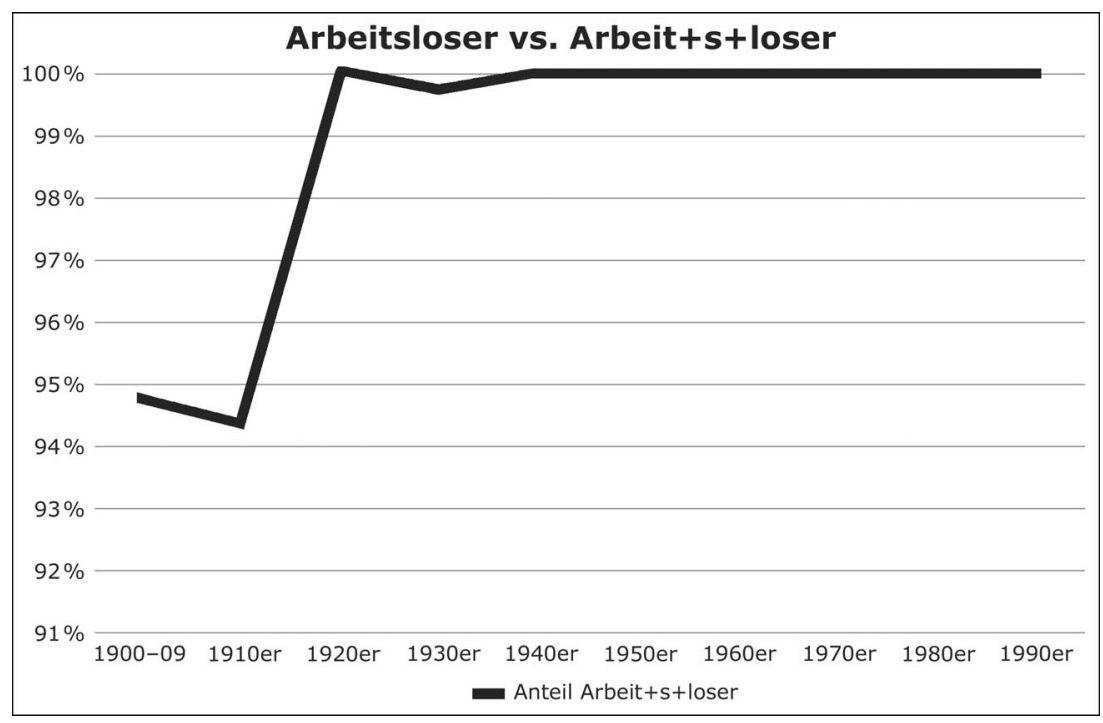

Diagramm 1: Die Entwicklung der s-Fuge bei Arbeit(s)loser (1.148 Treffer).

4. Ähnliches beschreibt Becker (1992: 11), ebenfalls basierend auf Wörterbuchanalysen. 


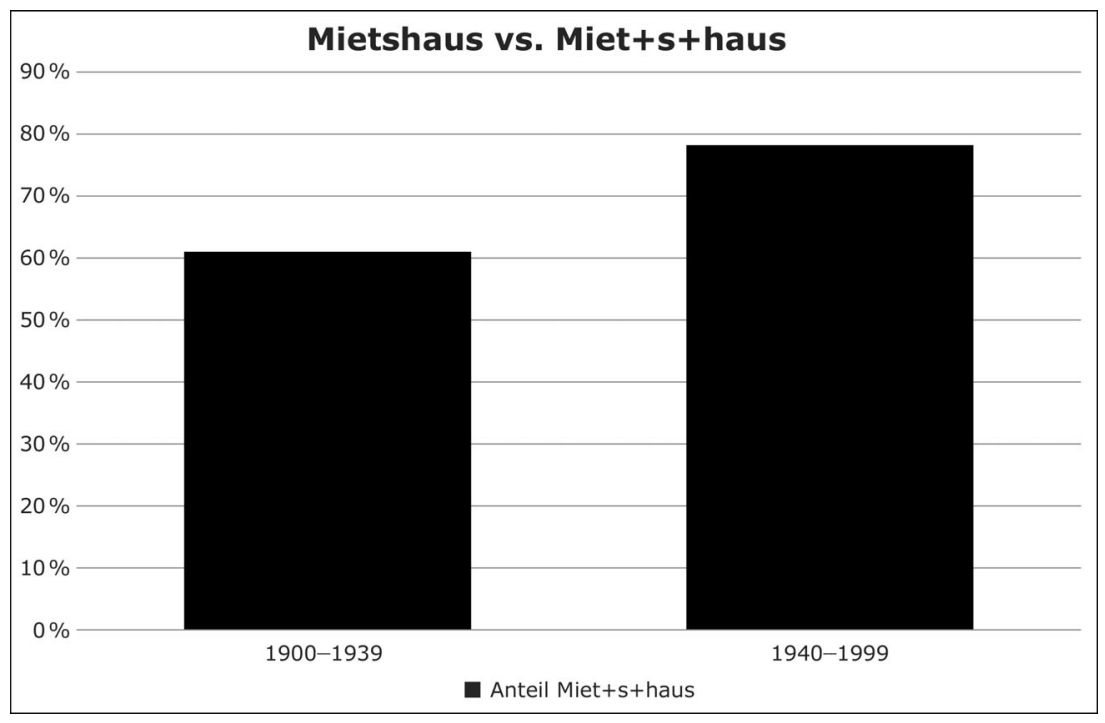

Diagramm 2: Die Entwicklung der s-Fuge bei Miet(s)haus (137 Treffer).

Diagramm 2 zeigt das Verhalten bei Miet(s)haus, wobei die Jahre 1900-1939 und 1940-1999 zusammenfasst wurden. Hier manifestiert sich der Umbruch. Insgesamt liegen 137 Komposita vor.

Auch für Diagramm 3 wurden einige Jahrzehnte zusammengefasst, um besser zu zeigen, wann sich der Umbruch hin zum Fugen-s ergibt. Dieser Abfrage liegen 256 Tokens zugrunde.

Somit gehen wir im Folgenden davon aus, dass das Deutsche derzeit eine Ausweitung der $s$-Verfugung erlebt und damit einen diachronen Trend fortsetzt. Andere Fugenelemente sind nicht annäherungsweise von solch starken Schwankungen betroffen.

In diesem Beitrag beschränken wir uns auf die $\mathrm{N}+\mathrm{N}-\mathrm{Komposita}$ (die $\mathrm{N}+\mathrm{A}$-Komposita scheinen sich jedoch ähnlich zu verhalten). Dabei soll der Frage nachgegangen werden, ob diese in Tabelle 1 und 2 aufgeführten über 60 Zweifelsfälle etwas Gemeinsames verbindet, das zu diesen Schwankungen Anlass geben könnte. Es wird zum einen dafür argumentiert, dass es primär phonologische Eigenschaften des Erstglieds sind, die die Verfugung steuern, und dass das Deutsche gegenwärtig einen tiefgreifenden fugengrammatischen Sprachwandel vollzieht. Die derzeitige Etappe dieses Wandels lässt sich präzise anhand dieser Schwankungsfälle bestimmen. Zum anderen wird der Frage nachgegangen, ob nicht auch das Zweitglied Einfluss auf die Verfugung ausübt. Es werden Argumente dafür geliefert, dass die $s$-Fuge ein Marker für Nominalität ist. Damit 


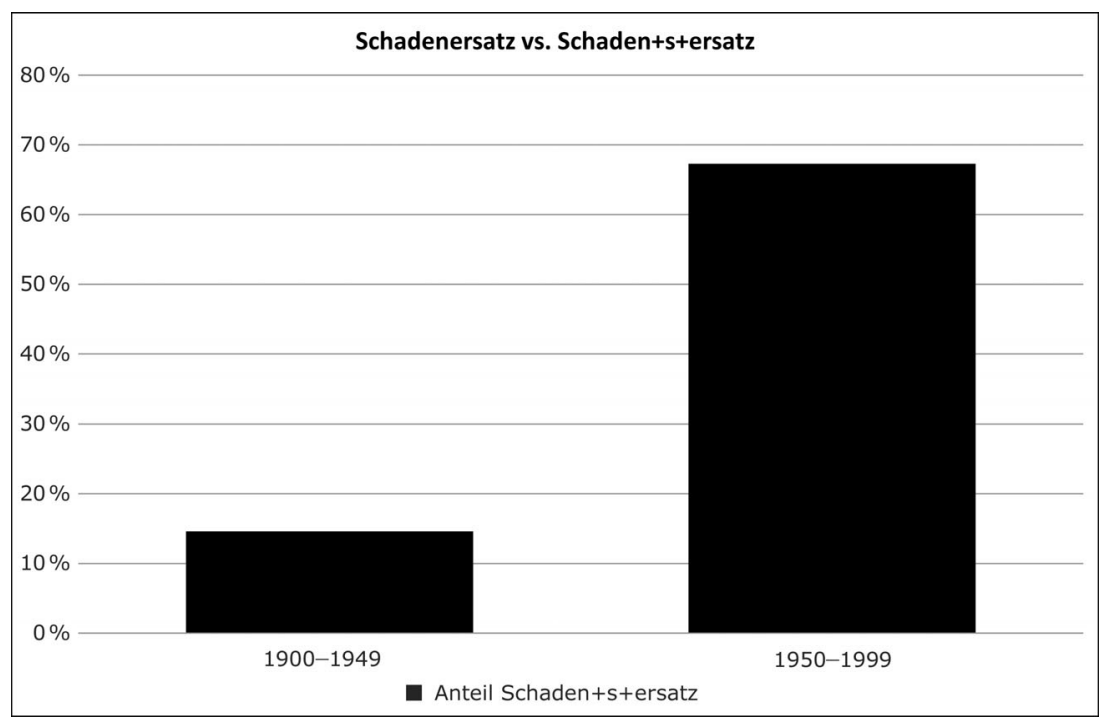

Diagramm 3: Die Entwicklung der s-Fuge bei Schaden(s)ersatz (256 Treffer).

wandelt sich die $s$-Fuge von einem einstigen morphologisch konditionierten Flexionssuffix im Frühnhd. zu einer zunehmend phonologisch konditionierten Grenzmarkierung mit der Funktion der Binnenstrukturierung von Komposita, in manchen Fällen sogar zur Anzeige von Nominalität.

\section{Die s-Verfugung}

Bevor wir speziell auf die $s$-Fuge eingehen, gilt es einige Präliminarien zu Fugenelementen abzuhandeln. Fremdfugen wie in Elektr+o+gerät bleiben hier unberücksichtigt.

\subsection{Fugenelemente im Allgemeinen}

Fugenelemente lassen sich als das phonologische Material ermitteln, das gegenüber der Nom.Sg.-Form hinzukommt, wenn ein Nomen zum Determinationsglied eines Kompositums wird: "Dabei wird als Fuge genau das angesehen, was über die Form des Nom Sg eines substantivischen Determinans hinausgeht" (Eisenberg 2006: 236), vgl. Frau - Frau+en+schuh, Rabe - Rabe+n+futter, Land - Land +es+sprache, Ausland Ausland $+s+j a h r$ etc. Ein und dasselbe Nomen kann zuweilen mehrere sog. Kompositionsstammformen ausbilden, vgl. Kind - Kind $+\varnothing+$ bett, Kind $+s+$ tod, Kind $+e s+$ missbrauch, Kind + er + wagen, ebenso Land $+\varnothing+-$ 
Tabelle 3: Häufigkeit und Art der Verfugung (nach Kürschner 2003).

\begin{tabular}{|l|l|}
\hline Fugenelement & prozentualer Anteil \\
\hline$-\varnothing-$ & $58 \%$ \\
\hline$-s-$ & $25 \%$ \\
\hline$-(e) n-$ & $11 \%$ \\
\hline$-e s-$ & $4 \%$ \\
\hline restliche & $2 \%$ \\
\hline
\end{tabular}

$e i$, Land $+s+$ mann, Land $+e s+k i n d$, Länd $+e r+$ beschreibung. ${ }^{5}$ Diese Fugen sind fest distribuiert und lassen sich folglich nicht austauschen: $*$ Kind $+s+$ wagen, *Kind + er + missbrauch etc. Der Normalfall bleibt jedoch die Nicht- oder Nullverfugung vom Typ Haustür, Türrahmen, Rahmenbedingung.

Nach Ortner et al. (1991: 69) hängt die Verfugungshäufigkeit von der Textsorte ab: In Erzählprosa und sog. Mischtexten wird zu 72,8\% nullverfugt, in wissenschaftlicher Prosa dagegen nur zu 58,8\%. Auf 58\% Nullfugensetzung kommt auch Kürschner (2003, 2005) in seinem zeitungssprachlichen Korpus. Darauf folgt gemäß Kürschner schon die $s$ Fuge mit $25 \%$, d.h. $-s$ - ist das mit Abstand häufigste und gleichzeitig das produktivste Fugenelement. Ortner et al. (1991) fassen die $s$ - und die (viel seltenere) es-Fuge leider zusammen und gelangen für die wissenschaftliche Prosa auf insgesamt 31,3\%. Dabei stehen $s$ - und es-Fuge nicht in komplementärer Distribution, wie dies für die $(e) n$-Fuge gilt und die für den Erhalt bzw. die Herstellung von Trochäen genutzt wird: Dame $+n+$ abteilung, aber Frau+en+bewegung. Outputorientiert unterstützt die (e)n-Fuge also die Herstellung der optimalen (d.h. trochäischen) phonologischen Wortform. Dieses komplementäre Verhalten ist gerade für Substantive der schwachen Deklination typisch und hier auch produktiv. So verwundert es nicht, dass die (e)n-Fuge nach Kürschner (2003, 2005) mit $11 \%$ das zweithäufigste Element bildet. Die verbleibenden $6 \%$ verteilen sich auf die restlichen Fugenelemente -es- (mit allein $4 \%$ ), -er-, -e-, -ens- und -ns-. Tabelle 3 liefert den Überblick.

Fugenelemente gelten grundsätzlich als Nichtkasusmarker (Gallmann 1998), auch wenn sie historisch aus Genitivkonstruktionen mit präpo-

5. Viele Artikel über Fugenelemente (so auch Becker 1992: 15) weisen auf den Sonderfall hin, dass die verschiedenen Kompositionsstammformen von Kind teilweise semantisch gesteuert sein könnten: Kind $+s+$ im medizinischen, Kind $+e s+$ im juristischen Bereich und Kind $+e r+$ im unmarkierten Fall. Hierbei handelt es sich um einen singulären Fall, der für ähnliche Kompositionsstammformvarianten nicht mehr gilt wie Mann $+s+$, Mann+es+, Männ $+e r+$ oder Land $+s+$, Land $+e s+, L a ̈ n d+e r+$. Ob die ersten beiden Kompositionsstammformen von Kind- überhaupt produktiv sind, ist ungeklärt. 
niertem Genitiv hervorgegangen sind: [[der Sonnen (Gen.Sg.)] Strahl] $>$ $[$ der $[$ Sonne $+n+$ strahl]]. Viele Sprecher wollen auch heute noch Fugenelemente durch Genitivparaphrasen erklärt wissen. $\mathrm{Zu}$ viele Beispiele sprechen jedoch dagegen, vgl. Liebling $+s+$ getränk, Bischof $+s+k o n f e-$ renz, Dreikönig $+s+$ tag oder Dieb $+e s+$ bande, deren Genitivlesart entweder unsinnig (*Getränk des Lieblings) oder an eine inkongruente Singularlesart gekoppelt wäre (*Konferenz des Bischofs). Kontroverser wird eine mögliche Pluralinformation diskutiert, die sich im Fall von Kind $+e r+$ wagen und Hühn+er+ei zwar verbietet, doch im Fall von Dubletten vom Typ Land+es+regierung vs. Länd + er + regierung oder Volk $+s+k u n d e$ vs. Völk $+e r+k u n d e$ naheliegend ist. Dies gilt insbesondere auch für schwache, finalbetonte Feminina vom Typ Geburt+en+kontrolle, Schrift + en + verzeichnis. ${ }^{6}$ Hierzu schreibt Fuhrhop (2000: 203):

Produktiv verwendet werden sie [hier: $-e r-$ und -e-; DN/RS] paradigmisch bei Pluralbedeutung wie Schilderwald (vs. Schildbürger), Ärztekongress (vs. Arztpraxis). Die 'Plural'-Interpretation ist zum Teil auch bei -en- möglich, insbesondere wenn es die unparadigmische $s$-Fuge 'ersetzt' wie in Antiquitätenhandel; dies erscheint jedoch als Sonderfall. ${ }^{7}$

Psycholinguistische Forschungen weisen dagegen eine Pluralbedeutung zurück (Dressler et al. 2001, Koester et al. 2004).

Zurecht mahnt Wegener (2005), dass sich viele Fugenelemente nur mit sprachhistorischen Kenntnissen verstehen lassen: "Die Fugenelemente sind ein Paradebeispiel dafür, dass man manchmal ohne historisches Wissen, ohne Kenntnis früherer Sprachstufen, nicht auskommt und zu hoffnungslosen Fehldeutungen verleitet wird" (Wegener 2005: 157). Sowohl bei Sonne $+n+$ strahl, Nase $+n+$ höhle als auch - nach Wegener bei Gäns $+e+k e u l e$, Rind $+e r+b r a t e n$ und Hühn $+e r+e i$ handelt es sich um frühere Gen.Sg.-Formen (vgl. noch ahd. gensi 'Gans' (Fem.Gen.Sg.), lembires 'Lamms' (Mask.Gen.Sg.)), wobei -ir- hier noch stammbildendes Suffix ist, das später einerseits zum Pluralsuffix, andererseits zum Fugenelement "grammatikalisiert" wurde (Wegener 2005; s. auch Fuhrhop 1996). Bis heute sind die -(e)n-, die -e- sowie die -er-Fugen (weitestgehend) an ihre alten Klassen gekoppelt. Dennoch ist nicht auszuschließen,

\footnotetext{
6. S. auch Becker (1992: 12). Im Fall von Müttergenesungsheim oder Töchterschule kann es sogar nur die Pluralbedeutung sein, die Mütter- (bzw. Töchter-) gegenüber Mutter(bzw. Tochter-) favorisieren lässt, da hier ein zusätzliches Fugenelement gar nicht hinzukommt.

7. Genau diesem Sonderfall entspricht unser Zweifelsfall Unterschrift(s/en)aktion.
} 
dass Sprecher heute eine Reanalyse dieser kategoriell entleerten Elemente vornehmen (s. Fuhrhop 1996: 544, 548; 2000: 204, sowie Fn. 2). ${ }^{8}$

Eine wichtige Unterscheidung betrifft die zwischen paradigmischen und unparadigmischen Fugenelementen (Ortner et al. 1991, Fuhrhop 1996, 1998, Eisenberg 2006): Paradigmische Fugenelemente entsprechen formal einer Flexionsform des Paradigmas, unparadigmische Fugenelemente tun dies nicht. So handelt es sich bei Frau+en+fußball um eine paradigmische Fuge, da Frauen im Flexionsparadigma von Frau vorkommt, nicht aber bei Storch+en+schnabel, da *Storchen keine Flexionsform von Storch bildet. Hier lautet der Gen.Sg. Storchs, der Pl. Störche. Historisch lässt sich jedoch erklären, weshalb es zu unparadigmischen en-Fugen kommt: Die betreffenden Substantive gehörten einst zur schwachen Klasse und sind in eine andere Klasse übergewechselt. Ihr Fugenverhalten hat sich dem jedoch nicht angepasst. Während nach Ortner et al. (1991: 76) die unparadigmischen en-Fugen (es gibt keine unparadigmischen $n$-Fugen) nur 3\% unter allen (e)n-Fugen ausmachen, also die Ausnahme bilden, verhält es sich bei der $s$-Fuge ganz anders: "Im ganzen übertrifft die Zahl der Komposita mit unparadigmischer - $s$ Fuge die Fälle mit paradigmischer" (Ortner et al. 1991: 76). Hier sind es vor allem die zahlreichen Feminina, die zu Buche schlagen und weder historisch noch heute ein $-s$ im Paradigma enthalten (von seltenen Fremdwörtern mit $s$-Plural abgesehen): Neben einigen Simplizia wie Liebe, Hilfe oder Arbeit (Liebe $+s+$ beweis, Hilf $+s+$ angebot, Arbeit $+s+-$ $a m t$ ) werden reihenweise, regulär und produktiv vor allem Derivate auf -ion, -ität, -heit/-(ig)keit, -schaft, -sal, -tum und -ung mit -s- verbunden. Dies treibt ihre Zahl in die Höhe, gerade in Fachtexten, weshalb hier die $s$-Fugenwerte höher sind (s. Kürschner 2003, 2005). Besonders häufig werden auch Präfigierungen wie Zufahrt $+s+s t r a ß e$, Unschuld $+s+$ miene, Vorsicht $+s+$ maßnahme mit $-s$ - verfugt. Damit wird deutlich: Während die unparadigmischen en-Fugen Zeugen früherer Flexionsklassenzugehörigkeit sind, unproduktiv sind und sich konservativ verhalten, haben sich die unparadigmischen $s$-Fugen genau umgekehrt stark ausgeweitet und aus ihren ursprünglichen Domänen gelöst. Schließlich sind sie auf die Feminina übergegangen, bei denen sie sich seither besonders stark ausbreiten.

Aus funktionaler Perspektive sind Fugenelemente als Grenzsignale innerhalb von Komposita zu verstehen. Sie signalisieren dem Hörer, dass eine Folge zweier (oder mehr) phonologischer Wörter als hierarchisch

8. Auf eine durchgehende naive Fehlinterpretation der Fugenelemente als Kasus- und Numerusmarker beruht der durch Jean Paul im frühen 19. Jh. vorgebrachte Aufruf, das "verfemte Zwischen=s" (so ironisierend von Briegleb 1935) ersatzlos zu streichen (als Reaktion auf Jean Paul, s. Grimm 1819 und Briegleb 1935). 
gestuftes Kompositum (Determinans - Determinatum) zu dekodieren ist. Dies heißt nicht, dass nicht weitere prosodische Informationen hinzukämen (z. B. der Kompositionsakzent). Mit den Fugenelementen liegt jedoch eine materielle Wortgrenzmarkierung vor, die sich zunehmend ausbreitet. Das Duden-Wörterbuch der sprachlichen Zweifelsfälle (2007) empfiehlt, dass im Fall eines Fugen- $s$ eine Bindestrichschreibung von Komposita entfällt: Das Fugenelement bindet Komposita damit auch auf der graphematischen Ebene stärker zusammen. Fuhrhop (2000) beantwortet die im Titel ihres Beitrags gestellte Frage, ob Fugenelemente die Morphologisierung von Komposita anzeigen, positiv: So finde durch den Verlust der ursprünglichen Genitivfunktion von -s- eine "Entsyntaktisierung" (Fuhrhop 2000: 204) statt wie in Richtung weisend > richtung $(+s+)$ weisend. Doch auch die Bildung von Kompositionsstammformen insbesondere durch unparadigmische (und damit eindeutige) Fugenelemente (wie in Freiheit+s+-, Versicherung $+s+-$ ) zeige die Morphologisierung an. Außerdem haben Aronoff \& Fuhrhop (2002) nachgewiesen, dass Fugenelemente derivationsmorphologisch geschlossene Stämme für die Komposition öffnen, so etwa bei -ung und -ling: *meinunglich, *Meinungchen etc., aber Meinung $+s+$ umfrage; *Prüflingin, $*$ Prüflingchen, aber Prüfling $+s+$ treffen.

\subsection{Die s-Fuge im Besonderen}

Zusammenfassend ist zu sagen, dass die $s$-Fuge das häufigste, das produktivste und das innovativste Fugenelement darstellt. Auch nichtnative Erstglieder werden häufig mit $-s$ - verfugt, regelmäßig die Fremdsuffixe -ion und -(i)tät. Die es-Fuge ist von alledem entkoppelt und "auf der Strecke geblieben", sie alterniert also nicht systematisch mit $-s-$. Beschränkt man sich nur auf die materiellen Elemente, rechnet man also die Nullfugen aus Tabelle 3 heraus, so ergibt sich, dass knapp $60 \%$ aller Fugenelemente auf $-s$ - entfallen, ca. $26 \%$ auf -(e)n-, 9,5\% auf $-e s-$ und $4,8 \%$ auf die verbleibenden. Wie bereits festgestellt, tritt $-s$ - öfter unparadigmisch als paradigmisch auf (-es- dagegen nur paradigmisch). Außerdem ist $-s$ - völlig frei von einer möglichen Plurallesart und relativ frei von einer Genitivinterpretation, in jedem Fall bei den zahlreichen Feminina. In gewisser Weise hat es sich am meisten von seinem Ursprung entfernt und erfüllt es am klarsten die bisher genannte Funktion der morphologischen Grenzanzeige.

Doch nicht nur das: Wenn Komposita an Komplexität zunehmen so der bisherige Forschungsstand -, dann steigt auch die Wahrscheinlichkeit einer $s$-Fuge, vgl. Sicht $+\varnothing+$ schutz - Absicht $+s+$ erklärung, Schuld $+\varnothing+$ frage - Unschuld $+s+$ miene, Nacht $+\varnothing+z u g-$ Weih$n a c h t+s+$ gans etc. Dies hat zu der Annahme geführt, dass Fugenele- 
mente auf morphologische Komplexität reagieren. Doch hat sich dies als unzureichend herausgestellt: Besteht nämlich das gesamte Erstglied aus einem Kompositum (Typ Bilderbuch-, Rückruf-), so nimmt, wie Kürschner (2003) ermittelt hat, deren $s$-Verfugung kaum zu (vgl. Bilderbuch $+\varnothing+$ wetter, Rückruf $+\varnothing+a k t i o n)$. Nur dann, wenn das Erstglied derivationell komplex ist (und auch hier nur unter spezifischen Bedingungen), schnellt die Verfugungshäufigkeit von $25 \%$ auf $67,5 \%$ nach oben (zuungunsten der Nullfuge, mit der die $s$-Fuge fast ausschließlich konkurriert): Beruf $+s+$ wunsch.

In Nübling \& Szczepaniak (2008) haben wir nachgewiesen, dass es nicht primär die morphologische Komplexität des Erstglieds, auch nicht die derivationelle, ist, sondern seine wortphonologische Qualität, die die Fugensetzung steuert: Derivationelle Komplexität generiert oft schlechte phonologische Wörter, die dann umso eher verfugt werden. So haben Derivationssuffixe wie -schaft, -heit oder -ung einen unklaren Status zwischen phonologischem Wort und Reduktionssilbe und werden daher durch das Fugen-s hervorgehoben (zum phonologischen Wort s. u. a. Hall 1999, Wiese 2000). Dabei gilt ganz pauschal: Besonders schlechte phonologische Wörter werden im Deutschen bereits zuverlässig s-verfugt und besonders gute phonologische Wörter am seltensten. Die zunehmende $s$ Verfugung hat also bereits diejenigen Wörter erfasst, die am weitesten vom phonologischen Wortideal, das Einsilber und trochäische Einfüßer mit Reduktionssilbe umfasst, entfernt sind. Dies sind z. B. Derivate mit unbetontem Präfix wie Berúf $+s+$, Verkáuf $+s+$, Entwúrf $+s+$, aber auch solche mit betontem Suffix Versícherùng $+s+$, Religión $+s+$, Érb-

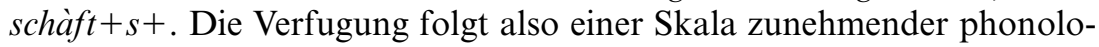
gischer Wortqualität. Dabei ist zu berücksichtigen, dass Fremdwörter häufig schlechte phonologische Wörter bilden (Nübling \& Szczepaniak 2009). Dies wird in Abschnitt 3 vertieft.

Eine weitere Besonderheit kennzeichnet die $s$-Fuge: Mit Verbinfinitiven homophone deverbale Nomen wie Essen, Leben, Wissen, Misstrauen bilden zwar von ihrer Struktur sehr gute phonologische Wörter, dennoch werden sie zuverlässig mit $-s$ - verfugt: Essen $+s+$ marke, Leben $+s+g e-$ $f a h r$, Wissen $+s+$ durst, Misstrauen $+s+$ votum. Mit der Verfugung werden diese Einheiten eindeutig als nominal ausgewiesen. Fuhrhop (1996: 535) zeigt außerdem, dass es sich häufig um untrennbare Präfixverben handelt und dass ihre Nominalisierung neutrales Genus besitzen muss (s. auch Briegleb 1935: 19).

Der einzige Einfluss, den ein Zweitglied auf die Verfugung auszuüben vermag, scheint seine ausgeprägte Verbalität zu sein: Insbesondere deverbale Abstraktbildungen wirken durch ihre noch vorhandene Argumentstruktur auf das Erstglied ein und blockieren dabei tendenziell seine Verfugung, vgl. Stellung $+\varnothing+$ nahme. Deutlich ist dies bei nominalisierten 
Infinitiven zu sehen, selbst wenn ihnen Erstglieder, die auf ein -s forderndes Suffix enden, vorangehen, vgl. das Zeitung $+\varnothing+$ lesen, aber die Zeitung $+s+$ lektüre. Hieraus resultieren einige Schwankungsfälle, z. B. die "offiziell" zwar unverfugten (realiter jedoch oft verfugten) Rektionskomposita wie Stellung $+\varnothing+$ nahme (dagegen: Stellung $+s+$ variante), Krieg $+\varnothing+$ führung (neben Krieg $+s+$ führung), Arbeit $+\varnothing+$ geber, Arbeit$+\varnothing+$ nehmer (dagegen: Arbeit $+s+$ wille), Gewicht $+\varnothing+$ heber (neben manchmal vorkommendem Gewicht $+s+$ heber, dagegen immer Gewicht $+s+$ probleme). Die $s$-Fuge bricht dieses Rektionsverhältnis auf und entsyntaktisiert bzw. morphologisiert dadurch das Kompositum. Es erhöht bzw. indiziert damit seine Nominalität. Gleiches gilt für deverbale Partizipkomposita vom Typ richtung $+\varnothing+$ weisend $>$ richtung $+s+-$ weisend, erfolg $+\emptyset+$ versprechend $>$ erfolg $+s+$ versprechend, Achtung gebietend $>$ achtung $+s+$ gebietend (Fuhrhop 1996).

\section{Der Sprachwandel}

Ausgehend von den konkreten Zweifelsfällen in Tabelle 1 und 2 zeichnen sich zwei Hauptfaktoren ab, die die Schwankungen bedingen: Zum einen sind es Komposita mit deverbalem Zweitglied, dessen Verbalität noch die alte Argumentstruktur transportiert. Dies verursacht den Schwankungstyp Stellung $(+s+)$ nahme, Auftrag $(+s+)$ geber, Antrag $(+s+)$ steller, Aufwand $(+s+)$ entwicklung, Schaden $(+s+)$ ersatz (s. Abschnitt 3.1). Zum anderen und in der großen Mehrzahl der Fälle geht die Schwankung vom Erstglied aus, wenn dieses aus einem nicht wohlgeformten phonologischen Wort besteht, das - um korrekt dekodiert zu werden durch das Fugen- $s$ als Grenzsignal an seinem Ende verstärkt wird. Hiervon zeugen Schwankungen vom Typ Merkmal $(+s+)$ analyse, Subjekt $(+s+)$ pronomen und Seminar $(+s+)$ arbeit (hierzu Abschnitt 3.2). Selbstverständlich können auch beide Faktoren gleichzeitig wirken, wie dies für Testament $(+s+)$ eröffnung und Knochenmark $(+s+)$ transplantation gelten dürfte.

\subsection{Die s-Verfugung als Nominalisierungsverstärker}

3.1.1. Infinitiv als Erstglied. Es scheint eine fast eherne Regel zu sein, nominalisierte Infinitive im Erstglied mit $-s-\mathrm{zu}$ verfugen: Wissen $+s+-$ durst, Glücken $+s+$ bedingung, Schaden $+s+$ begrenzung, Schaffen $+s+-$ kraft etc. Dies kontrastiert mit der unter 3.2 auszuführenden Tatsache, dass es sich bei Infinitiven wortphonologisch um gut erkennbare Wörter handelt, hier um Trochäen mit Reduktionssilbe. Genau solche Wörter bleiben üblicherweise unverfugt, da sie als Wort bestens erkennbar sind. Anders bei nominalisierten Infinitiven, die normalerweise sehr regelmäBig verfugt werden, gerade um nicht für einen Infinitiv gehalten zu wer- 
den. Nur Schaden $(+s+)$ ersatz und Essen $(+s+)$ ausgabe ${ }^{9}$ bilden Zweifelsfälle mit nominalisiertem Infinitiv als Erstglied, die mit $32 \%$ bzw. $34 \%$ Null- und mit $68 \%$ bzw. $66 \% s$-Fugensetzung stark schwanken. Doch dürfte die Ursache der Schwankung vom Zweitglied ausgehen, vor allem wenn man festverfugte Komposita wie Essen $+s+z e i t$, Essen $+s+-$ ration, Essen $+s+$ geruch, Essen $+s+$ gewohnheit danebenstellt. Im Fall von Schaden $(+s+)$ - wirkt auch die Fachsprachlichkeit ein. Der fugenhemmende Einfluss deverbaler Zweiglieder ist Gegenstand des nächsten Absatzes.

3.1.2. Hoher Verbalitätsgrad des Zweitglieds. An Beispielpaaren wie Zeitung $(+s+)$ lesen vs. Zeitung $+s+$ deutsch, Stellung $(+s+)$ nahme vs. Stellung $+s+$ variante, auch Rücksicht $+\emptyset+$ nahme vs. Rücksicht $+s+l o-$ sigkeit u.v. a.m. zeigt sich, dass (deverbale) Zweitglieder mit noch starkem verbalem Gehalt dazu tendieren, eine fugenhemmende Wirkung auszuüben und auf diese Weise Schwankungsfälle zu verursachen, selbst bei ansonsten obligatorisch verfugten Erstgliedern auf -ung. In den meisten Fällen hat sich - als Ausdruck der Verbalität - die alte Argumentstruktur konserviert, die vor allem darin besteht, ein direktes Objekt zu fordern. Dies erklärt die Schwankungen bzw. Unsicherheiten bei Auftrag $(+s+)$ geber, Aufwand $(+s+)$ entwicklung, Stellung $(+s+)$ nahme, Knochenmark $(+s+)$ transplantation, Gewicht $(+s+)$ heber, Essen $(+s+)-$ ausgabe, Testament $(+s+)$ eröffnung, Schaden $(+s+)$ ersatz: Das Erstglied wirkt unverfugt wie ein Akkusativ, mit $s$-Fuge dagegen verbietet sich die Annahme eines solchen Rektionsverhältnisses. ${ }^{10}$ Damit wirkt die $s$-Fuge, wie bereits gesagt, deverbalisierend bzw. nominalisierend sowie entsyntaktisierend bzw. morphologisierend. Da die aufbereiteten Korpora des IDS, des DWDS und von Wortschatz-Leipzig nur wenige Tokens pro Item ergeben, liegen der folgenden Tabelle sowie der Schematisierung in Abbildung 1 die viel zahlreicheren (aber auch fehlerträchtigeren) Google-Ergebnisse (vom 24. 08. 09) zugrunde. Tabelle 4 dokumentiert die Ergebnisse in absoluten und relativen Werten und unterscheidet dabei zwischen Büchern, die eher konzeptionell schriftlich sind, sowie Foren und Blogs. Auch wenn die Zahlen und Prozentwerte, die sich auf jeweils $+/$-verfugt beziehen, sehr unterschiedlich ausfallen, so handelt es sich doch, gemäß der eingangs zitierten Definition, um Zweifelsfälle.

9. Allerdings hat das Kompositum Essen $(+s+)$ ausgabe auch eine metonymische, nicht bzw. weniger verbale Lesart, nämlich als Ort, an dem das Essen ausgegeben oder durchgereicht wird.

10. Gerade von diesem Rektionskompositionstyp dürfte es viele weitere Schwankungsfälle geben, z. B. Abstand $(+s+)$ messer, Abstand $(+s+)$ halter etc. 
Tabelle 4: Rechercheergebnisse zu einigen Fugenschwankungsfällen aus verschiedenen Google-Domänen ${ }^{11}$ (weiß: unverfugt, grau: verfugt).

\begin{tabular}{|c|c|c|c|c|c|c|}
\hline Kompositum & Bücher & & Foren & & Blogs & \\
\hline Suchanfrage in "...” & absolut & $\%$ & absolut & $\%$ & absolut & $\%$ \\
\hline Zeitung $+\varnothing+$ lesen & 7.850 & 50,5 & 2.360 & 70 & 1.950 & 66,5 \\
\hline Zeitung $+s+$ lesen & 7.680 & 49,5 & 1.010 & 30 & 983 & 33,5 \\
\hline Stellung $+\varnothing+$ nahme & 569.000 & 98,8 & 70.100 & 90 & 68.100 & 94,5 \\
\hline Stellung $+s+$ nahme & 6.520 & 1,2 & 7.830 & 10 & 4.000 & $\overline{5,5}$ \\
\hline Rechnung $+\varnothing+$ stellung & 1.500 & 11,3 & 1.620 & 22 & 265 & 8 \\
\hline Rechnung $+s+$ stellung & 11.800 & 88,7 & 5.760 & 78 & 3.000 & 92 \\
\hline Krieg $+\varnothing+$ führung & 168.000 & 72,5 & 4.100 & 18 & 3.400 & 20,7 \\
\hline Krieg $+s+$ führung & 63.700 & 27,5 & 18.700 & 82 & 13.000 & 79,2 \\
\hline Essen $+\varnothing+$ ausgabe & 3.350 & 46,2 & 227 & 14 & 235 & 10 \\
\hline Essen $+s+$ ausgabe & 3.900 & 53,8 & 1.390 & 86 & 2.110 & 90 \\
\hline Gewicht $+\varnothing+$ heber & 3.700 & 97,2 & 2.890 & 97,9 & 3.290 & 98,8 \\
\hline Gewicht $+s+$ heber & 104 & 2,8 & 62 & 2,1 & 40 & 1,2 \\
\hline Arbeit $+\varnothing+$ geber & 357.000 & 97,8 & 181.000 & 97 & 65.400 & 97,2 \\
\hline Arbeit $+s+$ geber & 7.700 & 2,2 & 5.480 & 3 & 1.850 & 2,8 \\
\hline Arbeit $+\varnothing+$ nehmer & 431.000 & 99,5 & 95.000 & 98,9 & 47.200 & 98,5 \\
\hline Arbeit $+s+n e h m e r$ & 2.090 & 0,5 & 1.050 & 1,1 & 687 & $\overline{1,4}$ \\
\hline Auftrag $+\varnothing+$ geber & 202.000 & 99,5 & 40.400 & 96,3 & 40.600 & 98,4 \\
\hline Auftrag $+s+$ geber & 919 & 0,5 & 1.520 & 3,6 & 658 & 1,6 \\
\hline das Antrag+Ø+stellen & 56 & 100 & 15 & 100 & 5 & 100 \\
\hline das Antrags $+s+$ stellen & 0 & 0 & 0 & 0 & 0 & 0 \\
\hline Antrag $+\varnothing+$ stellung & 58.900 & 94 & 23.900 & 82,6 & 4.780 & 73,4 \\
\hline Antrag $+s+$ stellung & 3.780 & 6 & 5.030 & 17,4 & 1.730 & 26,6 \\
\hline Antrag $+\varnothing+$ steller & 126.000 & 97,8 & 18.200 & 87,4 & 7.380 & 80 \\
\hline Antrag $+s+$ steller & 2.750 & 2,1 & 2.620 & 12,6 & 1.770 & 20 \\
\hline der Antrag $+\varnothing+$ berechtigte & 33 & 3 & 3 & & 0 & \\
\hline der Antrag $+s+$ berechtigte & 1.090 & 97 & 41 & & 8 & \\
\hline Antrag $+\varnothing+$ frist & 38 & 0,6 & 3 & & 2 & \\
\hline Antrag $+s+$ frist & 6.500 & 99,4 & 1.180 & 100 & 854 & 100 \\
\hline insgesamt ohne $s$-Fuge & 1.128.427 & 94,2 & 439.818 & 89,5 & 242.607 & 88,8 \\
\hline insgesamt mit $s$-Fuge & 118.533 & 5,8 & 51.673 & 10,5 & 30.690 & 11,2 \\
\hline
\end{tabular}

11. Absolute Zahlen unter 4 wurden nicht in Prozente umgerechnet. 
$\mathrm{Zu}$ Tabelle 4: Deutlich tritt hervor, dass die konzeptionell eher mündlichen Domänen (Foren und Blogs) höhere Werte für die $s$-Verfugung aufweisen als die Bücher (s. z. B. Stellung $+s+n a h m e, K r i e g+s+$ führung, Essen $+s+$ ausgabe, Antrags $+s+$ stellung, Antrag $+s+$ steller etc.). In den letzten beiden Zeilen wurden, bei aller Unterschiedlichkeit in der Trefferzahl, sämtliche Zahlen jeweils addiert und ihre Prozentwerte ermittelt, um den Einfluss der natürlich nur indirekt zu ermittelnden Mündlichbzw. Schriftlichkeit auf die $s$-Fugensetzung greifbarer zu machen. Außerdem erweist sich, dass mit der Abnahme des verbalen Gehalts des Zweitglieds die $s$-Verfugung zunimmt. Dies wird in Abbildung 1 exemplarisch anhand der folgenden Komposita mit dem konstanten Erstglied Antraggezeigt. Die Prozentwerte sind hier gemittelt, d. h. es wird von den drei Domänen abgesehen (manchmal wurde bei der Recherche ein Artikel hinzugenommen, um falsche Belege auszuschließen). Die $s$-Fuge ist bei extrem verbalem Antrag $+\varnothing+$ stellen, auch Antrag $+\varnothing+$ stellung sowie dem Nomen agentis Antrag $+\varnothing+$ steller kaum gebräuchlich, nimmt aber jeweils leicht $\mathrm{zu}$ (etwas mehr noch bei Antrag $(+s+)$ stellung als bei Antrag $(+s+)$ steller $)$, während die Zahlen bei den stark nominalen Komposita der Antrag $+s+$ berechtigte, Antrag $+s+$ frist, -dauer, -formular in die andere Richtung kippen, d.h. hier dominiert stark die $s$-Verfugung (Tab. 5).

Tabelle 5: Nominalität und ihre Korrelation zur s-Verfugung.

\begin{tabular}{|l|l|l|}
\hline Kompositum & $s$-verfugt & unverfugt \\
\hline das Antrag $(+s ?+)$ stellen & $0 \%$ & $100 \%$ \\
\hline Antrag $(+s ?+)$ stellung & $10,7 \%$ & $89,3 \%$ \\
\hline Antrag $(+s ?+)$ steller & $4,5 \%$ & $95,5 \%$ \\
\hline Antrag $(+s ?+)$ stellerin & $4 \%$ & $96 \%$ \\
\hline der Antrag $(+s ?+)$ berechtigte & $97 \%$ & $3 \%$ \\
\hline Antrag $(+s ?+)$ frist, -dauer, -formular & $100 \%$ & $0 \%$ \\
\hline
\end{tabular}

Abbildung 1 ordnet diese Zweifels- bzw. auch eindeutigen Fälle von Tabelle 5 auf einer Nominalitätsskala an und zeigt deren Korrelation zur zunehmenden $s$-Verfugung (für andere Komposita wie Auftrag $+\varnothing+g e$ ben, Auftrag $+\varnothing+$ vergabe, Auftrag $+\varnothing+$ geber vs. Auftrag $+s+$ beginn, Auftrag $+s+$ dauer würde sich ein ähnliches Bild ergeben). 


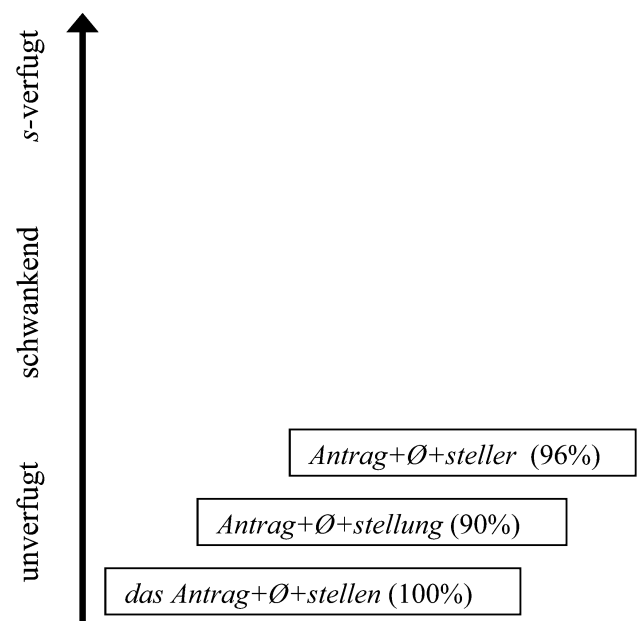

verbal
Antrag $+s+$ frist $(100 \%)$

Antrag + s + dauer $(100 \%)$

Antrag $+s+$ formular $(100 \%)$

der Antrag + s+berechtigte $(97 \%)$

Abbildung 1: Die Korrelation von Nominalität und s-Fuge.

\subsection{Die s-Verfugung als Indikator schlechter phonologischer Wortqualität}

Bis auf einige wenige Ausnahmen, die sich auf die bereits besprochene Funktion der $s$-Fuge als Nominalisierungsverstärker zurückführen lassen, ergeben sich die heutigen Zweifelsfälle in Tabelle 1 und 2 aus der prosodisch motivierten Expansion der $s$-Fuge. Dabei sind folgende prosodisch-phonologische Besonderheiten relevant:

1) Die Unabhängigkeit von der prosodischen Struktur des Erstglieds Im Gegensatz zu den restlichen Fugenelementen war die $s$-Fuge bereits in ihrer Entstehungszeit nicht an die prosodische Struktur des Basiswortes gebunden. Während andere Fugen sich bis heute an den trochäischen Outputstrukturen orientieren - eine Eigenschaft, die sie von ihren Quellen (den nominalen Flexionsendungen) geerbt haben - , ist die $s$-Fuge davon unabhängig, denn das Genitiv-s konnte schon seit dem Mittelhochdeutschen an ein- und mehrsilbige Stämme angehängt werden, z. B. mhd. des spils 'des Spiels', des faters 'des Vaters' (Szczepaniak 2010). Ähnlich wie die Flexionsbasis in Genitivformen muss auch das erste Kompositionsglied nicht trochäisch sein, um mit -s- verfugt zu werden, während schon die (viel seltenere und unproduktive) $e s$-Fuge nur an einsilbige Stämme treten kann und somit an den trochäischen Output gebunden ist (Land $+e s+$ museum, Bund $+e s+-$ anstalt). 
2) Die Verschlechterung der Silbenstruktur

Die $s$-Fuge trägt als unsilbisches Element stets zur Verschlechterung der Silbenstruktur bei: Im Gegensatz zu anderen unsilbischen Elementen, v. a. - $n$ - $($ Kunde $+n+$ dienst $)$, tritt $-s$ - gerade nicht an Vokale, sondern an Konsonanten heran, wodurch komplexe Silbenendränder entstehen. Die Tendenz zur Verschlechterung der Silbenstruktur beschränkt sich jedoch nicht auf die Bildung von Konsonantenclustern: Wie Wegener (2006) feststellt, ist die Frequenz der $s$-Fuge bei einsilbigen Erstgliedern höher, wenn diese auf die Obstruenten [p, t, k] auslauten, z. B. Ort $+s+$ tarif. In solchen Fällen bildet das Fugen- $s$ ein sog. extrasilbisches Element, da sein Sonoritätswert höher ist als der von Plosiven (Vennemann 1986: 36). Während solche Erstglieder häufig - bis zu $20 \%$ - s-verfugt werden, kommt die $s$-Fuge bei Einsilbern auf Sonoranten und Liquiden [m, n, l] nur bis zu 5\% vor, z. B. Heil $+s+$ lehre. Die $s$-Fuge tritt also tendenziell dort auf, wo sie dank ihrem extrasilbischen Charakter als Marker von Wortgrenzen besonders salient ist:

die $s$-Fuge nach auslautendem Plosiv (hohe Verfugungstendenz; 20\%) die $s$-Fuge nach auslautendem Liquid/Nasal (geringe Verfugungstendenz; 5\%)

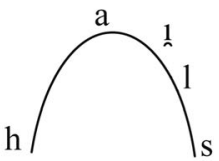

Heils-
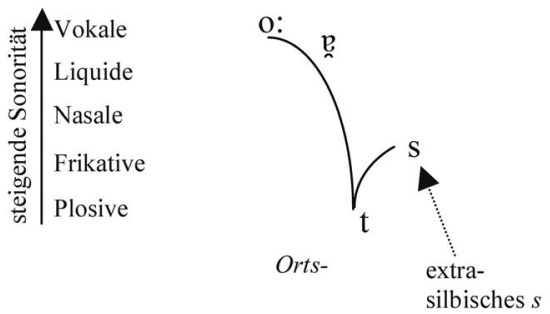

Abbildung 2: Das Fugen-s als Marker phonologischer Wortgrenzen.

Am Fugenverhalten der einsilbigen Erstglieder lässt sich also die prosodische Funktion der $s$-Fuge bereits deutlich erkennen. Sie markiert das Ende des ersten Kompositionsglieds, das zugleich ein separates phonologisches Wort $(\omega)$ darstellt, indem sie, überwiegend als extrasilbisches Element, an den Silbenendrand antritt wie in $[\text { Orts }]_{\omega}[\text { tarif }]_{\omega}$. Erstglieder wie Ort sind sowohl morphologisch als auch phonologisch einfach.

3.2.1. Phonologische Komplexität der Erstglieder. Nicht nur bei einfachen Erstgliedern ist das Auftreten der $s$-Fuge prosodisch gesteuert. Wie wir in Nübling \& Szczepaniak (2008) gezeigt haben, ist die Tendenz zur 
Tabelle 6: Die Häufigkeit des Fugen-s nach präfigierten Erstgliedern.

\begin{tabular}{|l|l|l|}
\hline & \multicolumn{2}{|l|}{ Erstglied enthält: } \\
\hline & $\begin{array}{l}\text { unbetontes Präfix } \\
\text { (Typ Beruf-) }\end{array}$ & $\begin{array}{l}\text { betontes Präfix } \\
\text { (Typ Anruf-) }\end{array}$ \\
\hline Tokens: & $85 \%$ (von 495.887 Komposita) & $36 \%$ (von 324.503 Komposita) \\
\hline Types: & $82 \%$ (von 17.999 Komposita) & $37 \%$ (von 11.325 Komposita) \\
\hline
\end{tabular}

$s$-Verfugung ebenfalls nach komplexen Erstgliedern von ihrer prosodischen Struktur abhängig. Während Kürschner (2003) die morphologische Komplexität als Steuerungsfaktor für die $s$-Fuge identifiziert hat, indem er anhand eines Zeitungskorpus bei derivationell komplexen Erstgliedern eine doppelt so hohe $s$-Verfugungsrate ermittelt hat wie bei kompositionell komplexen Erstgliedern, berücksichtigten wir die prosodische Struktur der morphologisch komplexen Glieder. Tabelle 6 zeigt, dass die Häufigkeit der $s$-Fuge bei Präfigierungen in unserem Korpus ${ }^{12}$ deutlich divergiert, je nachdem, ob das Präfix betont oder unbetont ist. Sie wird zu ca. $85 \%$ bei Derivaten mit unbetontem Präfix (Typ Berúf-) gesetzt. Erstglieder mit betontem Präfix (Typ Ánrüf) erhalten viel seltener ein Fugen$-s$, nämlich zu nur ca. $37 \%$, und damit fast genauso selten wie kompositionelle Erstglieder. Diese werden im Korpus von Kürschner (2003) zu nur $27 \% s$-verfugt. Die Häufigkeitsverhältnisse bleiben interessanterweise konstant, unabhängig davon, ob sie Types oder Tokens betreffen.

Die $s$-Verfugung variiert also je nach prosodischer Struktur der Präfigierungen, die im Folgenden im Rahmen der u. a. von Nespor \& Vogel (1986) vorgeschlagenen Theorie zur prosodischen Hierarchie vorgestellt wird. Unter Bezug auf die prosodische Struktur lassen sich die in Tabelle 1 und 2 aufgelisteten Zweifelsfälle adäquater erfassen, als wenn sie nur hinsichtlich der morphologischen Komplexität des Erstglieds untersucht würden. Tabelle 7 berücksichtigt nur die Zweifelsfälle, die ein Erbwort als Erstglied enthalten, und ordnet diese nach phonologischen und morphologischen Kriterien. Diese - zunächst hybride - Systematisierung wird im Folgenden einer ausschließlich phonologischen Analyse unterzogen (zu fremdsprachlichen Erstgliedern s. Abschnitt 3.2.2).

Die meisten Zweifelsfälle in Tabelle 7 betreffen solche Komposita, deren Erstglied ein Derivat mit betontem Präfix (z. B. Einkauf $(+s+)$ führer

12. Das untersuchte Korpus, das Archiv W der geschriebenen Korpora, enthielt zum Zeitpunkt der Analyse (März 2008) 1.105.545.477 Wortformen. Untersucht wurden alle Komposita, die im Erstglied eine von 40 ausgewählten Präfigierungen enthielten (19 mit unbetontem und 21 mit betontem Präfix). Zu Näherem s. Nübling \& Szczepaniak (2008: $18 \mathrm{f}$.$) .$ 


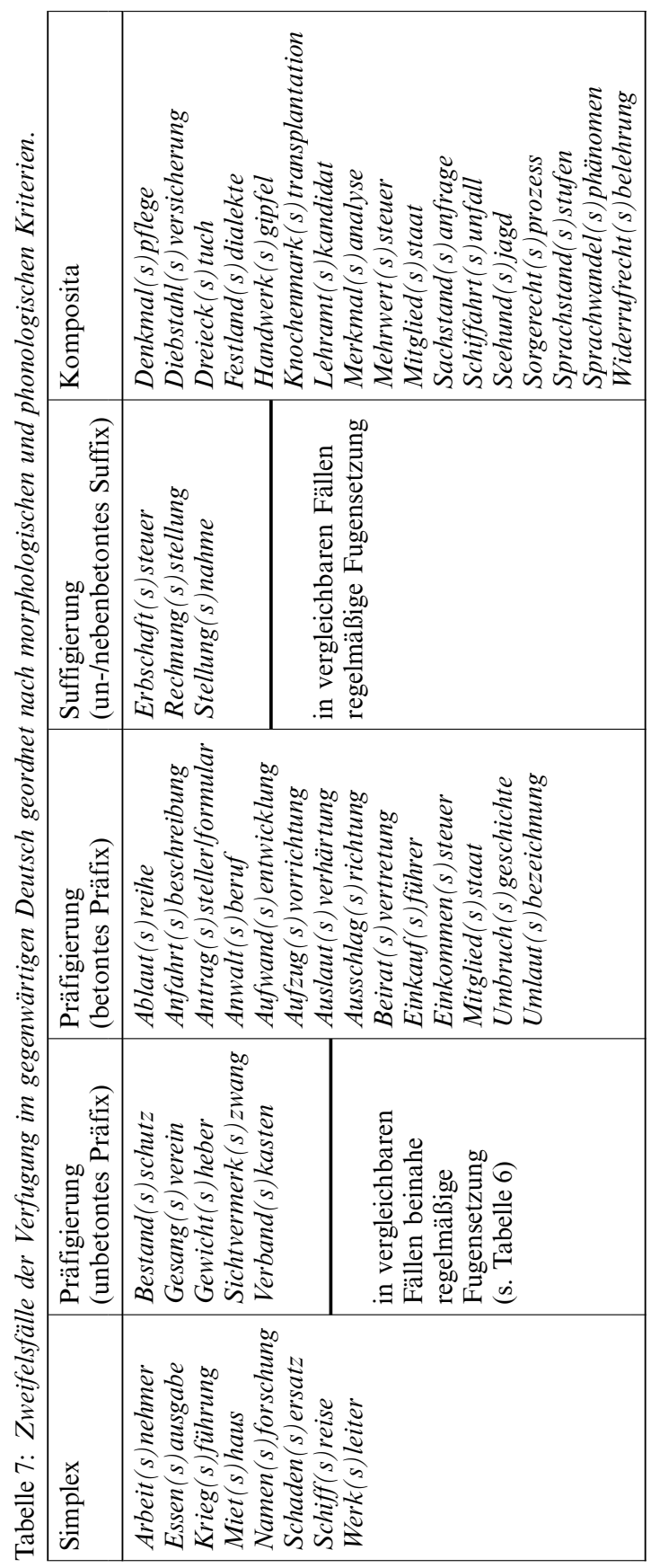


oder Mitglied $(+s+)$ staat) bzw. ein Kompositum ist (z. B. Festland $(+s+)$ dialekt oder Lehramt $(+s+)$ kandidat). In nur drei Fällen besteht das Erstglied in einer Suffigierung. Hier kommt die Fuge in vergleichbaren Fällen bereits regelmäßig vor. Der Schwankungsfall Erbschaft $(+s+)$ steuer ist auf die textsortenspezifischen Unterdrückung der $s$-Fuge v. a. in juristischen Texten zurückzuführen (s. Fn. 3). Die beiden übrigen Fälle Rechnung $(+s+)$ stellung und Stellung $(+s+)$ nahme sind mit der Verbalität des Zweitglieds zu erklären (s. Abschnitt 3.1). Diese spielt auch im Bereich der Komposita mit einem morphologisch einfachen Erstglied eine wichtige Rolle (Arbeit $(+s+)$ nehmer, Essen $(+s+)$ ausgabe, Krieg $(+s+)$ führung). Die nominalisierende und die prosodische Funktion der $s$-Fuge gehen hier Hand in Hand. Diese sowie die restlichen Fälle mit einfachem Erstglied enthalten entweder einen auf einen Obstruenten auslautenden Einsilber, der stark verfugt (z. B. Schiffs- oder Werks-), oder ein trochäisches Erstglied, dessen rechter Rand mit Hilfe von -s- prosodisch gestärkt wird (s. Abbildung 2). Diese Erstglieder (mit Ausnahme von Arbeit) sind phonologisch einfach, d. h. sie bilden ein einfaches phonologisches Wort $(\omega)$, z. B. [Namens $]_{\omega}[\text { forschung }]_{\omega}$. Insgesamt tritt die Verfugung bei phonologisch (und zugleich morphologisch) komplexen Erstgliedern häufiger auf.

Nur vereinzelt kommt es zu Schwankungsfällen bei Erstgliedern mit unbetontem Präfix. Dies ist damit verbunden, dass solche Komposita meist bereits regelmäßig (zu ca. $85 \%$ ) verfugt sind (s. Tabelle 6). Von den sechs Fällen, die in Tabelle 7 aufgelistet sind, treten zwei $(B e-$ stand $(+s+)$ schutz und Verband $(+s+)$ kasten) im Archiv W der geschriebenen Sprache des IDS überwiegend $s$-verfugt auf (s. Tabelle 8). Bei Gewicht $(+s+)$ heber wirkt die starke Verbalität des Zweitglieds der Verfugung entgegen. Die Verfugungsresistenz von Gesang könnte zum Teil phonologische Gründe haben: Der nasale Auslaut zieht die $s$-Fuge nicht so stark an wie der plosive wie z. B. in Bestand. Möglicherweise ist die

Tabelle 8: Die Häufigkeit der s-Verfugung bei den Zweifelsfällen mit unbetontem Präfix im Erstglied (Recherche im Archiv $W$ der geschriebenen Sprache des IDS; Suchanfrage vom 05. 08. 2009).

\begin{tabular}{|l|c|c|r|c|}
\hline Zweifelsfall & \multicolumn{2}{|c|}{ s-verfugt } & \multicolumn{2}{c|}{ unverfugt } \\
\hline & absolut & prozentual & absolut & prozentual \\
\hline Bestand $(+s+)$ schutz & 1186 & $90 \%$ & 130 & $10 \%$ \\
\hline Gesang $(+s+)$ verein & 2268 & $12 \%$ & 16039 & $88 \%$ \\
\hline Gewicht $(+s+)$ heber & 8 & $0,3 \%$ & 2610 & $99,7 \%$ \\
\hline Sichtvermerk $(+s+)$ zwang & 2 & $100 \%$ & 0 & \\
\hline Verband $(+s+)$ kasten & 602 & $81,7 \%$ & 135 & $18,3 \%$ \\
\hline
\end{tabular}


strukturelle Abweichung durch vorangestellte Reduktionssilbe (Typ Verfall)

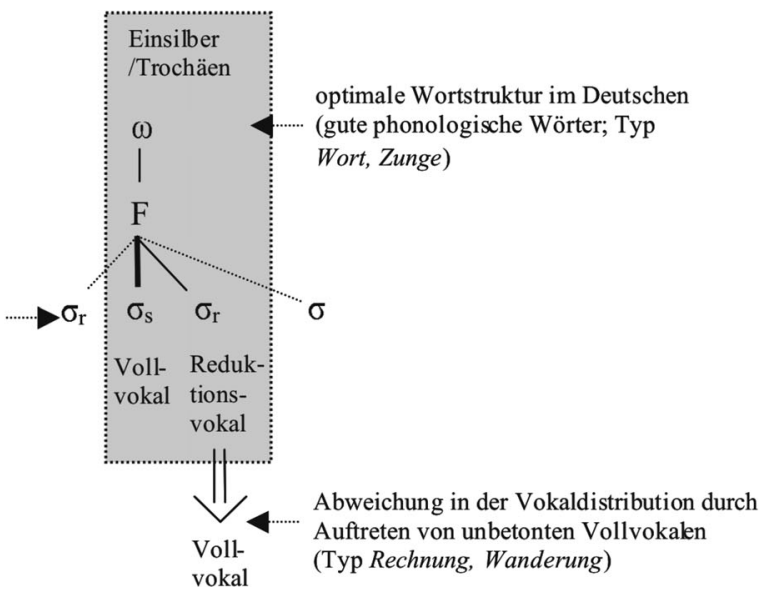

Abbildung 3: Das wortphonologische Ideal im Deutschen und mögliche Abweichungen.

Verfugungsblockade auch idiosynkratisch, d.h. lexembezogen. Die Schwankung bei Sichtvermerk $(+s+)$ zwang (nur zwei Belege im Korpus) könnte durch den textsortenspezifischen Gebrauch unverfugter Komposita bedingt sein.

Die heutigen Zweifelsfälle betreffen also hauptsächlich solche Komposita, die im Erstglied ein Derivat mit betontem Präfix oder ein Kompositum enthalten. Der heutige Stand der s-Verfugung ergibt sich daraus, dass sich die $s$-Fuge zunehmend zum phonologischen Mittel zur Hervorhebung des rechten Wortrands entwickelt hat: Sie tritt verstärkt an solche phonologischen Wörter, die vom wortphonologischen Ideal (Einsilber und Trochäen) abweichen (s. Abbildung 3). Diese bezeichnen wir als schlechte phonologische Wörter. Dazu gehören

1) Derivate mit unbetontem Präfix wie Verfáll,

2) Derivate mit dem Suffix -ung, das je nach prosodischer Struktur unbetont (Réchnung) oder nebenbetont (Wánderùngen) ist, sowie

3) Derivate mit nebenbetontem Suffix wie -schàft in Erbschàft.

Da sich die $s$-Fuge in Gruppe 2) und 3) fast vollständig durchgesetzt hat und auch in Gruppe 1) mit großer Regelmäßigkeit auftritt (ca. 85\%), gibt es in diesem Bereich wenige Zweifelsfälle (s. Abb. 4). Die prosodische Auffälligkeit von Gruppe 1) leitet sich von der Akzentstruktur ab (d. h. von der Prominenzstruktur innerhalb des Wortes): Das phonologische Wort beginnt nicht wie im trochäischen Optimalfall mit einer betonten Vollsilbe (vgl. Wolke), sondern mit einer Reduktionssilbe. Die Fälle 


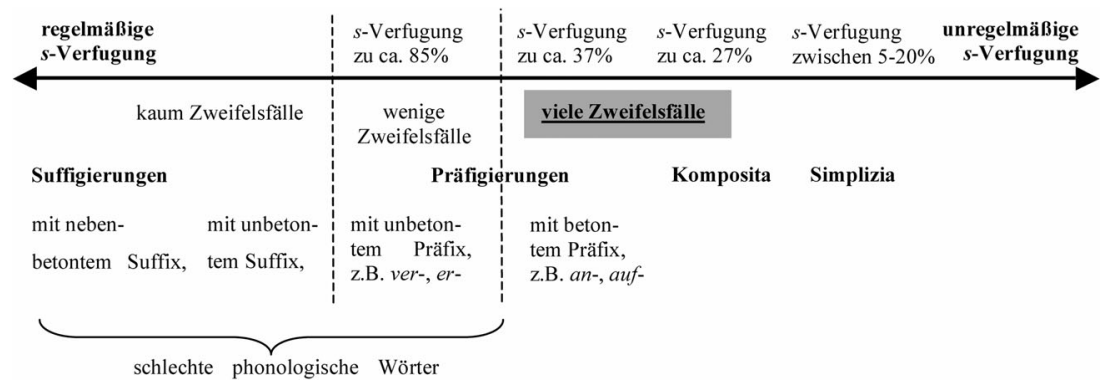

Abbildung 4: Die Korrelation von phonologischer Wortqualität und Verfugungsfrequenz.

unter 2) und 3) sind besonders interessant, da sie betonbare bzw. betonte Suffixe enthalten. In ung-Suffigierungen wird die Wortstruktur nicht wie im Optimalfall durch die Vokaldistribution hervorgehoben, vgl. ['vol.kə], ['le:.Rr]. Die ung-Suffigierungen enthalten in unbetonter Position keinen Schwalaut, sondern einen Vollvokal [U] wie in Rechnung ['REç.non] oder Wanderung ['van.də.Run]. Die nebenbetonten Suffixe wie -schaft oder -tum sind aufgrund ihrer prosodischen Eigenschaften als selbstständige phonologische Wörter zu betrachten: Sie enthalten einen Vollvokal, und ihr linker Rand stimmt (im Gegensatz zum -ung-Suffix) mit dem Silbenanfangsrand überein, vgl. Rechn+ung ['R\&ç.non] vs. Erb+schaft ['Eep. faft]. Dies berechtigt zu der Annahme, dass Derivate mit nebenbetonten Suffixen wie -schaft aus zwei phonologischen Wörtern bestehen $\left[\varepsilon \operatorname{rp}_{\omega}\left[\iint_{\mathrm{N}}\right]_{\omega}\right.$ (Raffelsiefen 2000). Die phonologische Wortstruktur solcher Suffigierungen unterscheidet sich also nicht von Komposita, die ebenfalls aus mindestens zwei phonologischen Wörtern bestehen, z. B. Tafel+obst ['ta:fəl $]_{\omega}[\mathrm{o}: p s t]_{\omega}$. Dass Suffixe wie -schaft einen anderen Status als Zweitglieder von Komposita haben, zeigt das Verhalten der $s$-Fuge, die nach diesen Suffixen obligatorisch ist. Wie wir in Nübling \& Szczepaniak (2009) argumentieren, ist die Verfugung an den besonderen phonologischen Status dieser Suffixe zurückzuführen: Sie bilden weniger prominente phonologische Wörter und werden deswegen mit der $s$-Fuge gestärkt. Diese phonologische Eigenschaft spiegelt sich in phonetischen Reduktionserscheinungen wider, z. B. Kürzung und gleichzeitiger Entspannung des $u$ in -tum: [Rarç.tu:m] > [Rarç.tom]. Die $s$-Fuge hat sich also zu einem Mittel entwickelt, mit dem schlechte phonologische Wörter optimiert werden. Dank ihrer phonologischen Salienz markiert die Fuge, häufig als extrasilbisches Element, den rechten Rand des letzten phonologischen Wortes im Erstglied und fördert hierdurch die hörerseitige Segmentierung der morphologischen Struktur der Komposita.

Während die schlechten phonologischen Wörter ziemlich regelmäßig eine Fuge zur Stärkung ihres rechten Randes enthalten, ist die Verfu- 
gungshäufigkeit nach Derivaten mit betontem Präfix (Anrùf+Ø+dienst) und nach Komposita (Nótrùf $+\varnothing+$ nummer) viel geringer. In diesen Fällen ist das letzte phonologische Wort (hier -rùf-) keinen Schwächungsprozessen ausgesetzt, da es auch nicht auf die weniger prominente (Nebenton-)Position beschränkt ist (vgl. der Ruf). Es ist interessanterund logischerweise der Typ Anrùf- und Nótrùf-, der die meisten Zweifelsfälle generiert. Nach demselben prosodischen Prinzip dringt die Fuge immer stärker in diese Bereiche vor, um das weniger prominente phonologische Wort hervorzuheben.

3.2.2. Fremdwort als Erstglied. Die wortoptimierende Funktion steuert auch die Ausdehnung der $s$-Fuge auf nichtnative Erstglieder. Unter den Zweifelsfällen in Tabelle 1 und 2 finden sich folgende:

Tabelle 9: Zweifelsfälle der Verfugung mit einem Fremdwort als Erstglied.

\begin{tabular}{|l|l|}
\hline 1. & Abitur $(s)$ feier \\
\hline 2. & Advent $(s)$ abend \\
\hline 3. & Datum $(s)$ angabe \\
\hline 4. & Diplom $(s)$ feier \\
\hline 5. & Interessen $(s)$ konflikt \\
\hline 6. & Latinum $(s)$ kurs \\
\hline 7. & Praktikum $(s)$ platz \\
\hline
\end{tabular}

\begin{tabular}{|r|l|}
\hline 8. & Präteritum $(s)$ schwund \\
\hline 9. & Referat $(s)$ besprechung \\
\hline 10. & Respekt $(s)$ person \\
\hline 11. & Seminar $(s)$ arbeit \\
\hline 12. & Subjekt $(s)$ pronomen \\
\hline 13. & Testament $(s)$ eröffnung \\
\hline
\end{tabular}

Die meisten Fremdwörter, v. a. solche romanischer oder griechischer Herkunft, weichen in ihrer prosodischen Struktur in mehreren Punkten vom Erbwortschatz ab (Näheres bei Nübling \& Szczepaniak 2009):

1) Sie enthalten Vollvokale auch in unbetonten Silben, wodurch der Vokalismus als Marker für die phonologische Wortstruktur entfällt, vgl. Diplom, Praktikum, Referat usw. Anders als bei Erb- und komplett assimilierten Lehnwörtern ist bei Fremdwörtern eine Korrelation zwischen Akzentstruktur und Vokalqualität nicht gegeben (s. Eisenberg 1991).

2) Fremdwörter haben eine sehr variable Akzentstruktur. Im Gegensatz zu trochäischen Erbwörtern lassen fremdsprachliche Simplizia auch den Finalakzent zu, z. B. Abitúr, Diplóm oder Advént (Becker 1996).

3) Fremdsuffixe tragen meist den Hauptakzent, wodurch sie sich von den nativen Suffixen, die entweder un- oder nebenbetont sind, unterscheiden, vgl. Réchn-ung (unbetont), Érb-schàft (nebenbetont), aber Rèlig-ión (hauptbetont). 
Diese Eigenschaften tragen dazu bei, dass viele Fremdwörter sehr schlechte phonologische Wörter bilden, die kaum zuverlässige Wortsignale (v. a. im Vokalismus) enthalten und vom einsilbigen bzw. trochäischen Wortideal stark abweichen (vgl. Abbildung 3). So ist die Ausweitung der $s$-Fuge auf nichtnative Kompositionserstglieder auf die Tendenz zur Markierung oder Optimierung der phonologischen Wortstrukturen zurückzuführen. Tatsächlich hat sich die $s$-Fuge bei einigen Fremdsuffixen, allen voran -ion und -(i)tät, bereits durchgesetzt, so dass diese heute immer verfugt werden, z. B. Universität $+s+$ gebäude, Religion $+s+-$ unterricht. Derzeit tendieren auch andere, ähnlich strukturierte Wörter zur $s$-Fuge: Es sind v. a. die Suffixe -(i)at, -ament/-ement und -um, die zunehmend verfugt werden (s. Nübling \& Szczepaniak 2009).

Unter den heutigen Zweifelsfällen in Tabelle 9 finden sich erwartungsgemäß gerade solche fremden Erstglieder, die 1) Finalakzent tragen (Abitur-, Advent-, Diplom-, Respekt-, Seminar- und Subjekt-13) und 2) das Suffix -um, -at oder -ament enthalten (Datum-, Latinum-, Praktikum-, Präteritum-, Referat-, Testament-). Interessen- [in.tR\&șən] weist durch die Vokaltilgung bereits eine trochäische Akzentstruktur auf. Die $s$-Fuge verstärkt wie in Essen $(+s+)$ marke den rechten Wortrand.

\section{Fazit: Die s-Fuge - ein Sprachwandelphänomen und seine Regularitäten}

Die heutigen Zweifelsfälle sind Ausdruck eines komplexen Sprachwandelphänomens. Sie enthalten fast ausschließlich die $s$-Fuge, die im Deutschen nicht nur das produktivste Fugenelement darstellt, sondern zugleich auch eins, das sich am weitesten von seinem flexivischen Ursprung entfernt hat. Neben der Tatsache, dass es die einzige produktive unparadigmische Fuge ist, die die morphologischen Schranken des früheren Flexivs durchbrochen hat, hat die $s$-Fuge neuartige Funktionen angenommen, die ihre heutige Ausbreitung steuern. Wie wir gezeigt haben, spiegeln die heutigen Zweifelsfälle zweierlei wider: Erstens die nominalisierende und zweitens die wortoptimierende Funktion der $s$-Fuge. So fördert die $s$-Fuge die Morphologisierung der Komposita, indem sie zum einen zur Unterdrückung der ursprünglichen Argumentstruktur in Komposita mit stark verbalem Zweitglied beiträgt (Krieg $+s+$ führung) und zum anderen regelmäßig nach nominalisierten Infinitiven im Erstglied auftritt (Wissen $+s+d u r s t$ ). Wortprosodisch hebt die Fuge das letzte Wort im Erstglied hervor, wenn seine Qualität nicht ausreichend ist bzw. von Schwächungsprozessen bedroht ist. Heute werden zunehmend auch

13. Die Betonung in Subjekt schwankt dabei ihrerseits zwischen Initial- und Finalakzent. 
wenig prominente (d.h. nebenbetonte) phonologische Wörter verfugt. Dies führt dazu, dass die heutigen Zweifelsfälle v. a. solche Komposita enthalten, die im Erstglied ein betontes Präfix (z. B. Einkauf $(+s+)$ führer oder Mitglied $(+s+)$ staat) bzw. ein Kompositum (z. B. Festland $(+s+)$ dialekt oder Lehramt $(+s+)$ kandidat) enthalten. Bei schlechteren wortphonologischen Verhältnissen erscheint die $s$-Fuge weitaus regelmäßiger.

Die komplexe Steuerung dieses Sprachwandelphänomens ist dafür verantwortlich, dass sich keine geradlinige, eindimensionale Entwicklung skizzieren lässt. Vielmehr sind hier auf fast allen sprachlichen Ebenen wirkende (semantische, morphologische und phonologische bis hin zu stilistischen) Steuerungsprinzipien identifiziert worden. Diese anhand gegenwärtiger Zweifelsfälle gewonnenen Erkenntnisse müssen in einem nächsten Schritt diachron empirisch überprüft werden.

Eingereicht: 10. 12. 2009

Überarbeite Fassung eingereicht:

11. 10. 2010
Deutsches Institut / Institut für Germanistik I Universität Mainz / Universität Hamburg nuebling@uni-mainz.de

Renata.Szczepaniak@uni-hamburg.de

\section{Literatur}

Aronoff, Mark \& Nanna Fuhrhop. 2002. Restricting suffix combinations in German and English: Closing suffixes and the monosuffix constraint. Natural Language and Linguistic Theory 20. 451-490.

Augst, Gerhard. 1975. Über das Fugenmorphem bei Zusammensetzungen. In Gerhard Augst (ed.), Untersuchungen zum Morpheminventar der deutschen Gegenwartssprache, 71-155. Tübingen: Narr.

Baufeld, Christa. 1996. Kleines frühneuhochdeutsches Wörterbuch. Lexik aus Dichtung und Fachliteratur des Frühneuhochdeutschen. Tübingen: Niemeyer.

Becker, Thomas. 1992. Compounding in German. Rivista di Linguistica 4 (1). 5-36.

Becker, Thomas. 1996. Die Aufhebung des Vokallängengegensatzes in unbetonten Silben der deutschen Standardsprache. Deutsche Sprache 24 (3). 268-282.

Brendler, Silvio. 2004. Namenarten und ihre Erforschung: Einleitung. In Andrea Brendler \& Silvio Brendler (eds.), Namenarten und ihre Erforschung. Ein Lehrbuch für das Studium der Onomastik, 33-48. Hamburg: Baar.

Briegleb, Otto. 1935. Das verfemte Zwischen $=s$ der Wortszusammensetzung. Leipzig: Friedrich Brandstetter.

Dressler, Wolfgang U., Gary Libben, Jacqueline Stark, Christiane Pons \& Gonia Jarema. 2001. The processing of interfixed German compounds. In Geert Booij \& Jaap van Marle (eds.), Yearbook of morphology 1999, 185-220. Dordrecht: Kluwer.

Duden - Richtiges und gutes Deutsch. Wörterbuch der sprachlichen Zweifelsfälle. 2007. Mannheim: Dudenverlag.

Eisenberg, Peter. 1991. Syllabische Struktur und Wortakzent: Prinzipien der Prosodik deutscher Wörter. Zeitschrift für Sprachwissenschaft 10 (1). 37-64.

Eisenberg, Peter. 2006. Grundriss der deutschen Grammatik, vol. 1. Stuttgart: Metzler.

Frilling, Sabine. 2004. Die Sprachberatung der GfdS. Resultate einer aktuellen Untersuchung. Der Sprachdienst 2. 43-49.

Frilling, Sabine. 2005. Die Sprachberatung der GfdS (II). Auswertung der Anfragen von November 2004 bis Januar 2005. Der Sprachdienst, 2-3. 69-77. 
Fuhrhop, Nanna. 1996. Fugenelemente. In Ewald Lang \& Gisela Zifonun (eds.), Deutsch typologisch, 525-550. Berlin: de Gruyter.

Fuhrhop, Nanna. 1998. Grenzfälle morphologischer Einheiten. Tübingen: StauffenburgVerlag.

Fuhrhop, Nanna. 2000. Zeigen Fugenelemente die Morphologisierung von Komposita an? In Rolf Thieroff, Matthias Tamrat, Nanna Fuhrhop \& Oliver Teuber (eds.), Deutsche Grammatik in Theorie und Praxis, 201-213. Tübingen: Niemeyer.

Gallmann, Peter. 1998. Fugenmorpheme als Nicht-Kasus-Morpheme. In Matthias Butt \& Nanna Fuhrhop (eds.), Variation und Stabilität in der Wortstruktur, 177-190. Hildesheim, Zürich \& New York: Georg Olms Verlag.

Grimm, Jacob. 1819. Jean Pauls neuliche Vorschläge, die Zusammensetzung der deutschen Substantive betreffend. Hermes 1819, vol. 2. 27-33.

Hall, Tracy Alan. 1999. The phonological word: a review. In Ursula Kleinhenz (eds.), Studies on the phonological word, 1-22. Amsterdam Philadelphia: Benjamins.

Klein, Wolf Peter. 2003. Sprachliche Zweifelsfälle als linguistischer Gegenstand. Zur Einführung in ein vergessenes Thema der Sprachwissenschaft. Linguistik Online 16 (4). 5-33.

Koester, Dirk, Thomas Gunter, Susanne Wagner \& Angela Friederici. 2004. Morphosyntax, prosody, and linking elements: The auditory processing of German nominal compounds. Journal of Cognitive Neuroscience 16 (9). 1647-1668.

Kürschner, Sebastian. 2003. Fugenelemente im Deutschen und Dänischen - eine kontrastive Studie zu einem Grenzfall der Morphologie. http://www.freidok.uni-freiburg.de/volltexte/ 1256 (21. 01. 2011).

Kürschner, Sebastian. 2005. Verfugung-s-nutzung kontrastiv: Zur Funktion der Fugenelemente im Deutschen und Dänischen. TijdSchrift voor Skandinavistiek, 26 (2). 101-125.

Mackowiak, Klaus \& Karin Steffen. 1991. Statistische Auswertung der Anfragen an das Grammatische Telefon. Diskussion Deutsch 22. 518-535.

Michel, Sascha. 2009. Schaden-0-ersatz vs. Schaden-s-ersatz. Ein Erklärungsansatz synchroner Schwankungsfälle bei der Fugenbildung von N+N-Komposita. Deutsche Sprache 4. $334-351$.

Michel, Sascha. 2010. Or+en+wurm, tag $+s+$ brief, kelb + er + arzet. Fugenelemente in N+NKomposita des Frühneuhochdeutschen. Beiträge zur Geschichte der deutschen Sprache und Literatur 132 (2). 177-199.

Nespor, Marina \& Irene Vogel. 1986. Prosodic Phonology. Dordrecht: Foris.

Nübling, Damaris. 2004. Vom Name-n-forscher zum Name-ns-forscher: Unbefugte oder befugte ns-Fuge in Namen(s)-Komposita? In Václav Bok, Ulla Williams \& Werner Williams-Krapp (eds.), Studien zur deutschen Sprache und Literatur. Festschrift für Konrad Kunze, 334-353. Hamburg: Verlag Dr. Kovac.

Nübling, Damaris \& Renata Szczepaniak. 2008. On the way from morphology to phonology: German linking elements and the role of the phonological word. Morphology 18. $1-25$.

Nübling, Damaris \& Renata Szczepaniak. 2009. Religion $+s+$ freiheit, Stabilität $+s+$ pakt und Subjekt $(+s+)$-pronomen: Fugenelemente als Marker phonologischer Wortgrenzen. In Peter Otto Müller (ed.), Studien zur Fremdwortbildung, 195-222. Hildesheim, Zürich \& New York: Georg Olms Verlag.

Ortner, Lorelies, Elgin Müller-Bollhagen, Hanspeter Ortner, Hans Wellmann, Maria Pümpel-Mader \& Hildegard Gärtner. 1991. Deutsche Wortbildung. Typen und Tendenzen in der Gegenwartssprache, vol. 4. Berlin \& New York: de Gruyter.

Raffelsiefen, Renate. 2000. Evidence for word-internal phonological words in German. In Rolf Thieroff, Matthias Tamrat, Nanna Fuhrhop \& Oliver Teuber (eds.), Deutsche Grammatik in Theorie und Praxis, 43-66. Tübingen: Niemeyer.

Strobel, Thomas. 2008. Die Sprachberatung der GfdS (III). Auswertung der telefonischen Anfragen von Januar bis Juni 2007. Der Sprachdienst 1. 31-42. 
Szczepaniak, Renata. 2010. Während des Flug(e)s/des Ausflug(e)s? German short and long genitive endings between norm and variation. In Alexandra Lenz \& Albrecht Plewnia (eds.), Grammar Between Norm and Variation, 103-126. Frankfurt: Peter Lang.

Vennemann, Theo. 1986. Neuere Entwicklungen in der Phonologie. Berlin \& New York: de Gruyter.

Wegener, Heide. 2005. Das Hühnerei vor der Hundehütte. Von der Notwendigkeit historischen Wissens in der Grammatikographie des Deutschen. In Elisabeth Berner, Manuela Böhm \& Anja Voeste (eds.), Ein gross und narhafft haffen. Festschrift für Joachim Gessinger, 157-169. Potsdam: Universitätsverlag.

Wegener, Heide. 2006. Statistical evidence for the role of phonology in the distribution and motivation of the linking element -s- in German. In Stephan Kepser \& Marga Reis (eds.), Pre-proceedings of the international conference on linguistic evidence: Empirical, theoretical and computational perspectives, 201-203. Tübingen: SFB 441.

Wiese, Richard. 2000. The phonology of German. 2. Auflage. Oxford: Clarendon Press. 